\title{
Quantum Mechanics of In Situ Synthesis of Metal Nanoparticles within Anionic Microgels
}

\author{
Mirza Wasif Baig and Muhammad Siddiq \\ Polymer Chemistry Laboratory 40, Department of Chemistry, Quaid-i-Azam University, Islamabad 45320, Pakistan \\ Correspondence should be addressed to Muhammad Siddiq; m_sidiq12@yahoo.com
}

Received 5 June 2013; Accepted 1 December 2013

Academic Editors: A. M. Lamsabhi and J. R. Sabin

Copyright ( 2013 M. W. Baig and M. Siddiq. This is an open access article distributed under the Creative Commons Attribution License, which permits unrestricted use, distribution, and reproduction in any medium, provided the original work is properly cited.

\begin{abstract}
We discuss the quantum mechanics of many-body systems, that is, hybrid microgel consisting of negatively charged anionic microgels possessing thick sheath of water molecules solvating protruding anionic moieties and nanoparticle captivated within the microgel. Thermodynamic feasibility of synthesis of particular nanoparticle within the microgel is dependent upon the magnitude of interaction between nanoparticle, water molecules, and microgel relative to sum of magnitude of self-interaction between counterions and interaction between counterions and microgel. Nanoparticles synthesized with in the microgels have thick electronic cloud that oscillates under the influence of net interaction potential of charged anionic moieties and solvent water molecules which constitutes the chemical environment of hybrid microgel. Hamiltonian describing energy of oscillating electronic cloud of wrapped around nanoparticle is mathematically derived to be equal to product of integral electron density and product of its position vector overall space and net force acting on the oscillating electronic cloud of nanoparticle is mathematically defined as; $\mathscr{F} \int \rho_{n}\{n\} \widehat{n} d n$.
\end{abstract}

\section{Introduction}

Metal nanoparticles encompass vast portion of nanomaterials. Synthesis of nanoparticles constitutes a crucial aspect of nanochemistry. Several strategies have been employed for the synthesis of nanoparticles of different dimensionalities [1]. The controllable synthesis is attained in the presence of suitable surfactants, templates, and capping agents such as polymers, ligands, and dendrimers [2]. Application of nanoparticles ranges from energy to medicine [3]. Optical properties of nanoparticles can be tuned by their size and shape which directly influence nanoscale excitons. Optical properties of NPS found that their noticeable applications are related to include light emitting devices, lasers, photovoltaics, detectors, and biolabels [4]. The application of nanoparticles in medicine has given birth to nanobiotechnology. Nanoparticles have the ability to penetrate the cell wall and deliver drugs or biomolecules into living systems for a therapeutic purpose [5]. For the last decade synthesis of nanoparticles in side polymer microgels have gained much attention. Template-based synthesis of nanoparticles in the interior of the microspheres is an alternative and effective approach for the synthesis of semiconductor, metal, and magnetic particles [6]. Karg and Hellweg have written a self-explanatory review about use of poly ( $\mathrm{N}$-isopropyl-acrylamide) as nanoreactor for synthesis of metal nanoparticles [7]. Use of anionic microgels as microreactor has been proved to be one of the most successful chemical methods for size and shape controlled synthesis of metal nanoparticles [8]. Microgels are synthesized by free radical emulsion polymerization of NIPAM with one or two appropriate monomers with anionic groups (normally carboxylic acid and sulphonic acid moieties) and methylene bisacrylamide as cross-linking agent. Microgel is a three-dimensional network with meshes in it possessing protruding anionic moieties that hold up water molecules and that are responsible for the swelling and deswelling of behavior of microgels [9]. Mesh present in typical microgel molecule can be shown in Figure 1.

Microgels provide a very handy method for the synthesis of nanoparticles at room temperature. Mesh present in microgels acts as microreactor for synthesis of nanoparticles. Size of mesh and number of charged anionic groups protruding in it together dictate the size and morphology of nanoparticles. The $\mathrm{pH}$ of external media controls the ionization of anionic 


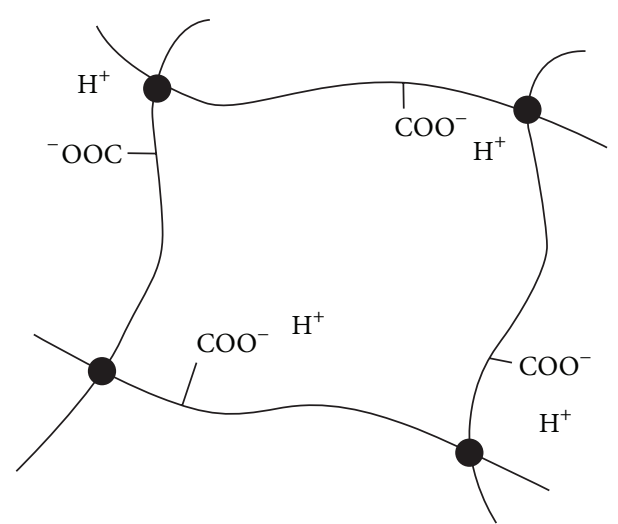

FIgURE 1: Structure of a mesh in an anionic microgel.

groups and also plays a key role in dictating the size and shape of nanoparticles. In aqueous solution of microgels solution metal salt of known concentration is added and stirred for a reasonable time to achieve complete homogeneity. Suitable reducing agent normally $\mathrm{NaBH}_{4}$ is added that reduces the metal cations having positive reduction potential or having reduction potential less negative than reducing agent that reduces them to neutral atoms. In other words, thermodynamically feasible reduction can be carried out with in microgel. Atoms reduced coagulate to form nanoparticles whose size is dictated by the above mentioned factors. Nanoparticles are of size exceeding the size of mesh in which they are produced this results in their long lasting captivity within the respective mesh of the microgel. Protruding anionic groups which initially attracted metal cations within the mesh are now in position to repel the oscillating electronic cloud present at the surface of nanoparticle which is responsible for its optical and catalytic properties. This repulsion is overcome by development of thick sheath of water molecules that solvates the captivated nanoparticles. Solvation is in such a way that bipolar solvent molecules orient one of their partial positively charged end towards the electronic sheath of nanoparticles and other end towards the negatively charged end of anionic moieties of microgels, thus stabilizing the charge repulsion and hence stabilizing the system. Microgels permanently captivate nanoparticles with in it and thick sheath of water molecules solvates them. Nanoparticles captivated with in the mesh of microgel can be shown in Figure 2.

\section{Theoretical Model}

According to best of our knowledge, physics behind the in situ synthesis of nanoparticles within the microgel has not been reported yet. Here we formulate quantum mechanics behind this in situ synthesis of nanoparticles within the microgel. Let us initially consider microgels to be dispersed in aqueous phase which is devoid of any other counter ions just possessing hydrogen ions produced from the ionization of anionic groups (normally carboxylic acid and sulphonic acid moieties) present in the microgels. Let us assume microgels possessing $N_{m}^{-}$anionic moieties giving up $N_{\mathrm{H}^{+}}^{+}$hydrogen

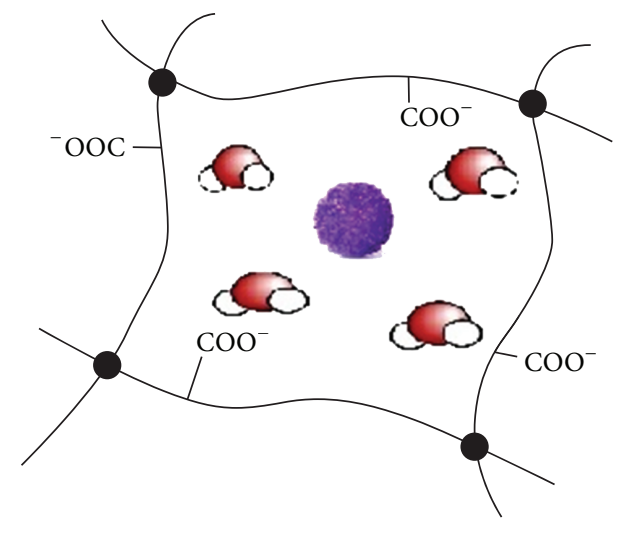

FIGURE 2: Structure of a mesh of hybrid anionic microgel captivating a nanoparticle.

ions. Adding solution of metal salt MX in microgel solution produces $N_{\mathrm{M}}^{+}$cations and anions $N_{\mathrm{X}}^{-}$. Electrical neutrality is achieved within the microgel by $N_{m}^{-}=N_{\mathrm{M}}^{+}$and outside the microgel by $N_{\mathrm{H}^{+}}^{+}=N_{\mathrm{X}}^{-}$. Interaction of microgels and counter metal cations can be mathematically modeled by the following total Hamiltonian [10]:

$$
H=H_{m}(\{x\})+H_{c}(\{y\})+H_{m c}(\{x\},\{y\}) .
$$

Hamiltonian $H_{m}$ for microgel is described in terms of kinetic energy and potential energy terms. First term is kinetic energy term and second term is potential energy term which explains potential of interaction among different microgel negatively charged moieties described by coordinates $x$. Hamiltonian for microgel is written as $[11,12]$

$$
H_{m}=K_{m}+\frac{1}{2} \sum_{i \neq j=1}^{N_{m}} v\left(\left\lfloor x_{i}-x_{j}\right\rfloor\right) \text {. }
$$

Hamiltonian $H_{c}$ for counter ions is described in terms of kinetic energy and potential energy terms. First term is kinetic energy term and second term is potential energy term which explains potential of interaction among different counterions described by coordinates $y$. Hamiltonian for counter ion is written as [10]

$$
H_{c}=K_{c}+\frac{1}{2} \sum_{i \neq j=1}^{N_{c}} v\left(\left\lfloor y_{i}-y_{j}\right\rfloor\right) .
$$

Hamiltonian $H_{m c}$ is written in terms of potential energy term and describes potential produced due to attractive interaction between counter ions and negative moieties of microgels and is mathematically expressed as

$$
H_{m c}=\sum_{i \neq j=1}^{N_{m}} \sum_{i \neq j=1}^{N_{c}} v\left(\left\lfloor x_{i}-y_{j}\right\rfloor\right) \text {. }
$$

Hamiltonian $H_{m c}$ in terms of density operators of microgel and density operators of counter ion can be written in following integral form:

$$
H_{m c}=\int \rho_{m}\{x\} d x \int \rho_{c}\{y\} d y,
$$


where density operators for microgel and counter ion can be defined as [12]

$$
\begin{aligned}
& \rho_{m}\{x\}=\sum_{j=1}^{N_{m}} \delta\left(x-x_{j}\right), \\
& \rho_{c}\{x\}=\sum_{j=1}^{N_{c}} \delta\left(y-y_{j}\right) .
\end{aligned}
$$

The mixture of microgels and counterions can be expressed by one component Hamiltonian by tracing coordinates of counter ion and partition function for counterions can be expressed as

$$
Z_{c}=\exp \left[\frac{-H_{c}+H_{m c}}{k_{B} T}\right] .
$$

Free energy associated with counterions can be expressed in terms of partition function as;

$$
G_{c}=-k_{B} T \ln \left(\exp \left[\frac{-H_{c}+H_{m c}}{k_{B} T}\right]\right) .
$$

When suitable reducing agent is added in solution of microgels and counterions, they get reduced and neutral atoms start agglomerating to form nanoparticles whose size is greater than the size of mesh and thus they are captivated within the respective mesh, where reduction is carried out. Mostly single nanoparticle is formed within one mesh of microgel. Nanoparticle is formed by aggregation of hundreds to thousands of atoms with oscillating electronic sheath around them. Let on reduction of metal cations captivated with in the meshes of microgels produce metal nanoparticle $M_{P}$ is formed with in the mesh. Now negatively charged groups normally carboxylic acid groups or sulphonic acid groups will not own this neutral nanoparticle wrapped with negatively charged electronic sheath. Thus, thick sheath of water molecules is sandwiched between electronic sheath of nanoparticle and protruding negatively charged moieties with in the mesh captivating the nanoparticle. Interaction between microgel negatively charged groups, strictly that holds water sheath and nanoparticles can be mathematically modeled by the following total Hamiltonian:

$$
\begin{aligned}
H= & H_{n}(\{n\})+H_{m}(\{x\})+H_{w}(\{z\})+H_{w n}(\{z\},\{n\}) \\
& +H_{w m}(\{z\},\{x\})+H_{m n}(\{x\},\{n\}) .
\end{aligned}
$$

Hamiltonian of nanoparticle described by coordinate $n$ is just composed of kinetic energy term as it can be regarded as a single particle captivated with in a particular mesh of microgel and thus it can be mathematically expressed as

$$
H_{n}(\{n\})=K_{n} \text {. }
$$

The previous Hamiltonian describing kinetic energy of nanoparticle can be approximated as zero because nanoparticle captivated in a mesh is composed of hundreds of atoms that hardly execute any translational motion but simply rotational motion which is too slow and ignorable and this term can be discarded; that is, $H_{n}(\{n\}) \cong 0$.

Hamiltonian $H_{w}$ for thick sheath of water molecules sandwiched between the negatively charged moieties of microgel and nanoparticle is described in terms of kinetic energy and potential energy terms. First term is kinetic energy term and second term is potential energy term which explains potential of interaction among different water molecules of thick sheath embedded between neutral nanoparticles and negatively charged moieties of the mesh described by coordinates $z$. Hamiltonian for microgel is written as

$$
H_{w}=K_{w}+\frac{1}{2} \sum_{i \neq j=1}^{N_{w}} v\left(\left\lfloor z_{i}-z_{j}\right\rfloor\right) .
$$

Hamiltonian $H_{w m}$ is written in terms of potential energy term and describes potential produced due to attractive interaction between water molecules and negatively charged moieties of microgel and is mathematically expressed as

$$
H_{w m}=\sum_{i \neq j=1}^{N_{w}} \sum_{i \neq j=1}^{N_{m}} v\left(\left\lfloor z_{i}-x_{j}\right\rfloor\right) .
$$

Hamiltonian $H_{w m}$ in terms of density operators of water and density operators of microgel can be written in the following integral form:

$$
H_{w m}=\int \rho_{w}\{z\} d z \int \rho_{m}\{y\} d y
$$

where density operators for water and nanoparticle can be defined as

$$
\begin{aligned}
& \rho_{w}\{z\}=\sum_{j=1}^{N_{w}} \delta\left(z-z_{j}\right), \\
& \rho_{m}\{x\}=\sum_{j=1}^{N_{m}} \delta\left(x-x_{j}\right) .
\end{aligned}
$$

Hamiltonian $H_{w n}$ can be expressed only in terms of potential energy term produced due to attractive interaction between water molecules and neutral nanoparticle present with in the microgel.

Consider

$$
H_{w n}=\int \rho_{n}\{n\} v(n) d n
$$

Potential energy originates due to attractive interaction between water molecules and neutral nanoparticle; it is between positively charged ends of water molecules with the electronic sheath wrapped around the nanoparticle. Oscillating electronic sheath wrapped around nanoparticle and several bounded metallic nuclei comprising nanoparticle can be regarded as a vibrating body. Electronic sheath is under the influence of electric field of these several bounded nuclei with a measurable force constant $k$. Nuclei comprising nanoparticles can be approximated as stationary with respect 
to oscillating electronic cloud; this approximation is on behalf of difference of mass of electronic cloud and nuclei. When thick sheath of water molecules surrounds the captivated nanoparticle then electronic sheath comes under the influence of electric field generated by oriented positively charged poles of thick sheath of water molecules. Net electric field $\xi_{w}$ generated by oriented positively charged poles of water molecules polarize the electronic cloud of nanoparticle and induces the dipole moment in it. Thus, Hamiltonian $H_{w c}$ in terms of dipole moment induced by electric field of water molecules can be mathematically expressed as

$$
H_{w n}=-\xi_{w} \int \rho_{n}\{n\} \widehat{\mu}(n) d n .
$$

Since dipole moment operator is product of position vector of electronic sheath and total charge it possesses and thus Hamiltonian $H_{w c}$ can be formulated as;

$$
H_{w n}=-\xi_{w} P e \int \rho_{n}\{n\} \widehat{n} d n .
$$

Hamiltonian $H_{m n}$ is expressed in terms of potential energy term and describes potential produced due to interaction between negatively charged moieties of microgels and neutral nanoparticle.

Consider

$$
H_{m n}=\int \rho_{n}\{n\} v(n) d n .
$$

Interaction between nanoparticles and microgel can be totally explained by interaction between protruding negatively charged anionic moieties (carboxylic acid groups and sulphonic acid groups) and wrapped electronic sheath along the periphery of nanoparticle. Electric field of anionic moieties $\xi_{a}$ also polarizes the electronic sheath by repulsive interaction inducing an electric field opposite to that induced by electric field of sheath of solvating water molecules. Thus $H_{m n}$ is mathematically expressed as

$$
H_{m n}=\xi_{a} \int \rho_{n}\{n\} \widehat{\mu}(n) d n .
$$

Since dipole moment operator is product of position vector of electronic sheath and total charge and thus Hamiltonian $H_{w c}$ can be formulated as;

$$
H_{m n}=\xi_{a} P e \int \rho_{n}\{n\} n d n
$$

The mixture of microgels, water, and nanoparticle can be expressed by one component Hamiltonian by tracing coordinate of nanoparticle and then partition function for nanoparticle can be expressed as

$$
Z_{n}=\exp \left[-H_{w n}+\frac{H_{m n}}{k_{B} T}\right] .
$$

Free energy associated with nanoparticle can be expressed in terms of partition function as

$$
G_{n}=-k_{B} T \ln \left(\exp \left[-H_{w n}+\frac{H_{m n}}{k_{B} T}\right]\right) .
$$

When in situ reduction of metal counterions present with in the meshes of microgels is carried out to produce their respective nanoparticle then free energy change accompanied with this reduction can be expressed as;

$$
\Delta G_{\text {in situ }}=G_{n}-G_{c} .
$$

Substituting free energies of counter ions and nanoparticles gives the following relation:

$$
\begin{aligned}
\Delta G_{\text {insitured }}= & -k_{B} T \ln \left(\exp \left[-H_{w n}+\frac{H_{m n}}{k_{B} T}\right]\right) \\
& +k_{B} T \ln \left(\exp \left[-H_{c}+\frac{H_{m c}}{k_{B} T}\right]\right) .
\end{aligned}
$$

Then in situ reduction will be spontaneous and exergonic; that is,

$$
\Delta G_{\text {insitu }}<0
$$

From (24) it can be mathematically interpreted that $\Delta G_{\text {insitured }}<0$ if

$$
H_{w n}+H_{m n}>H_{c}+H_{m c}
$$

Net Hamiltonian can also be written for describing interaction between anionic moieties of microgels and thick sheath of solvated water molecules and can be mathematically expressed as

$$
H_{\text {net }}=H_{w n}+H_{m n}=\left(\xi_{a}-\xi_{w}\right) P e \int \rho_{n}\{n\} \widehat{n} d n .
$$

From (27) it is evident that if electric field of water molecules, $\xi_{w}$, is greater in magnitude than that of electric field generated by anionic moieties, $\xi_{a}$, then value of net Hamiltonian will be attractive in nature and captivity of nanoparticle with in a mesh will be energetically feasible. Electric force acting on the electronic sheath of nanoparticle $\mathscr{F}$ can be set equal to product of net electric field within the mesh and charge possessed by electronic sheath of nanoparticle, that is, $\left(\xi_{a}-\xi_{w}\right) P e$ and thus $H_{\text {net }}$ transform as

$$
H_{\text {net }}=H_{w n}+H_{m n}=\mathscr{F} \int \rho_{n}\{n\} \widehat{n} d n
$$

\section{Discussion}

Initially water molecules are being attracted within a mesh by force of attraction with anionic moieties and counter ions with in the mesh after carrying out reduction with in mesh of microgel. They also get engaged in developing interaction with electronic cloud of the nanoparticle and overcome repulsive interaction between anionic moieties of gel and oscillating electric cloud of nanoparticle. Interaction between solvent water molecules and charge cloud of nanoparticle results in entrance of more water molecules inside the mesh followed by development of thick sheath of solvated water molecules within the mesh around the captivated nanoparticle. Electric fields are set up across the electronic cloud of 
nanoparticle by protruding anionic moieties of microgel and solvent water molecules with in the microgel that governs its swelling and deswelling behavior. Now electronic sheath wrapping up the nanoparticle oscillates in presence of an additional electric field set up by anionic moieties of microgel and solvent water molecules.

Net electric field appreciably polarizes the electronic cloud of nanoparticles inducing a dipole moment in it. Hamiltonian describing interaction between nanoparticle, microgel and water molecules is defined in terms of this induced dipole moment set up in the presence of net electric field. Magnitude of this interaction is directly related to the polarization behavior of nature of element under study. So soft metals that provide enough flexibility to their electronic cloud to get polarized under the influence of applied electric field could hold the induce dipole for a pretty measurable time. Thus, metal atoms with low ionization potential and high polarizability that can show affinity for sheath of water molecules solvating them leads to thermodynamically feasible captivity of nanoparticles. Thermodynamics of in situ reduction is totally dictated by this interaction. If eigenvalue of Hamiltonian describing interaction between induced dipole moment in electronic cloud of nanoparticle is greater than eigenvalue of Hamiltonians defining interaction between counter ions, water molecules, and microgels, then reduction becomes thermodynamically feasible. Magnitude of free energy is evaluated by difference of respective partition function. If electric field of solvated water particles is greater than electric field of anionic groups of microgels, then electronic cloud develops attractive interaction and dipole induced governs its various behavior including red shift in its surface plasmon band. This interaction between induced dipole of electric cloud and net electric field also contributes majorly towards encapsulation of nanoparticles with in the microgel. Encapsulation of nanoparticles with in a microgel is so strong that it strongly tunes its catalytic properties, as it hampers the upcoming substrate molecules toward nanoparticle and thus also reducing its catalytic activity [13].

\section{Conclusion}

Exergonicity of the in situ reduction of metal cations with in anionic microgel is dictated by the strength of interaction of net electric field generated by protruding anionic moieties of microgel and nanoparticle. So extent of polarization of electronic cloud is crucial for the thermodynamics of synthesis of nanoparticle with in a microgel. Magnitude of energy of interaction between nanoparticle and microgel can be obtained by product of integral of electron density and its position vector over all space multiplied with net force acting on the oscillating electronic cloud of nanoparticle, that is, $\mathscr{F} \int \rho_{n}\{n\} \widehat{n} d n$. Magnitude of this integral defines the fate of synthesis of nanoparticle if its magnitude exceeds, then the eigenvalue of Hamiltonians describes interaction between counter cations with water and microgel, and then reduction becomes thermodynamically feasible.

\section{Acknowledgment}

The authors acknowledge Professor Dr. Muhammad Ishaq for teaching advanced quantum chemistry course. The authors really admire his teaching skills and admit him to be one of best teachers the Chemistry department ever have.

\section{References}

[1] C. N. R. Rao, H. S. S. Ramakrishna Matte, R. Voggu, and A. Govindaraj, "Recent progress in the synthesis of inorganic nanoparticles," Dalton Transactions, vol. 41, no. 17, pp. 50895120, 2012.

[2] C. Altavilla and C. Ciliberto, Inorganic Nanoparticles, Taylor \& Francis, London, UK, 2011.

[3] S. E. Lohse and C. J. Murphy, "Applications of colloidal inorganic nanoparticles: from medicine to energy ," Journal of the American Chemical Society, vol. 134, 3, no. 8, pp. 15607-15620, 2012.

[4] G. D. Scholes, "Controlling the optical properties of inorganic nanoparticles," Advanced Functional Materials, vol. 18, no. 8, pp. 1157-1172, 2008.

[5] V. Sokolova and M. Epple, "Inorganic nanoparticles as carriers of nucleic acids into cells," Angewandte Chemie International Edition, vol. 47, no. 8, pp. 1382-1395, 2008.

[6] N. Welsch, M. Ballauff, and Y. Lu, "hermosensitive core-shell microgels: from colloidal model systems to nanoreactors," Progress in Polymer Science, vol. 36, no. 6, pp. 767-792, 2011.

[7] M. Karg and T. Hellweg, "Smart inorganic/organic hybrid microgels: synthesis and characterisation," Journal of Materials Chemistry, vol. 19, no. 46, pp. 8714-8727, 2009.

[8] J. Zhang, S. Xu, and E. Kumacheva, "Polymer microgels: reactors for semiconductor, metal, and magnetic nanoparticles," Journal of the American Chemical Society, vol. 126, no. 25, pp. 7908-7914, 2004.

[9] J. Zhang, L. Chu, Y. Li, and Y. M. Lee, "Dual thermo- and pHsensitive poly(N-isopropylacrylamide-co-acrylic acid) hydrogels with rapid response behaviors," Polymer, vol. 48, no. 6, pp. 1718-1728, 2007.

[10] A. R. Denton, "Effective interactions and volume energies in charged colloids: linear response theory," Physical Review E, vol. 62, no. 3, pp. 3855-3864, 2000.

[11] A. R. Denton, "Effective interactions and volume energies in charge-stabilized colloidal suspensions," Journal of Physics Condensed Matter, vol. 11, no. 50, pp. 10061-10071, 1999.

[12] A. R. Denton, "Counterion penetration and effective electrostatic interactions in solutions of polyelectrolyte stars and microgels," Physical Review E, vol. 67, no. 1, Article ID 011804, 2003.

[13] Y. Mei, Y. Lu, F. Polzer, and M. Ballauff, "Catalytic activity of palladium nanoparticles encapsulated in spherical polyelectrolyte brushes and core-shell microgels," Chemistry of Materials, vol. 19, no. 5, pp. 1062-1069, 2007. 

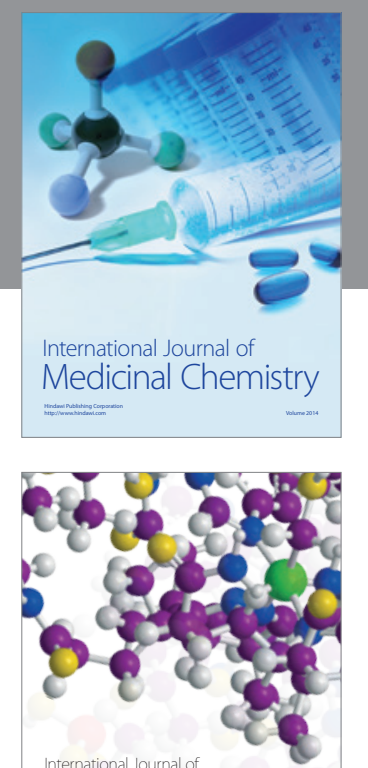

\section{Carbohydrate} Chemistry

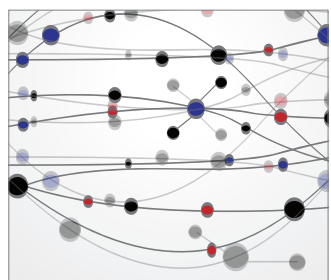

The Scientific World Journal
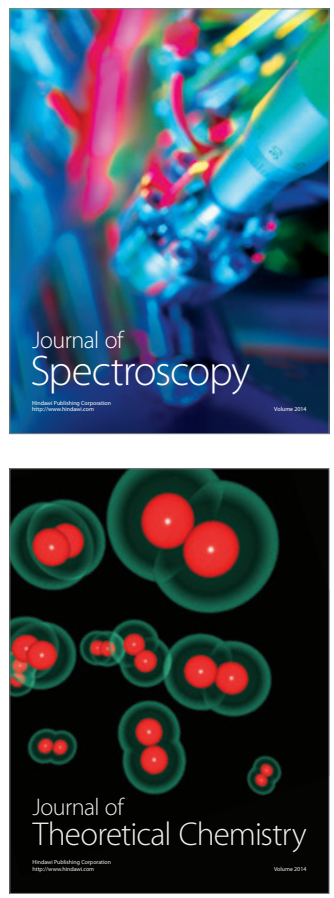
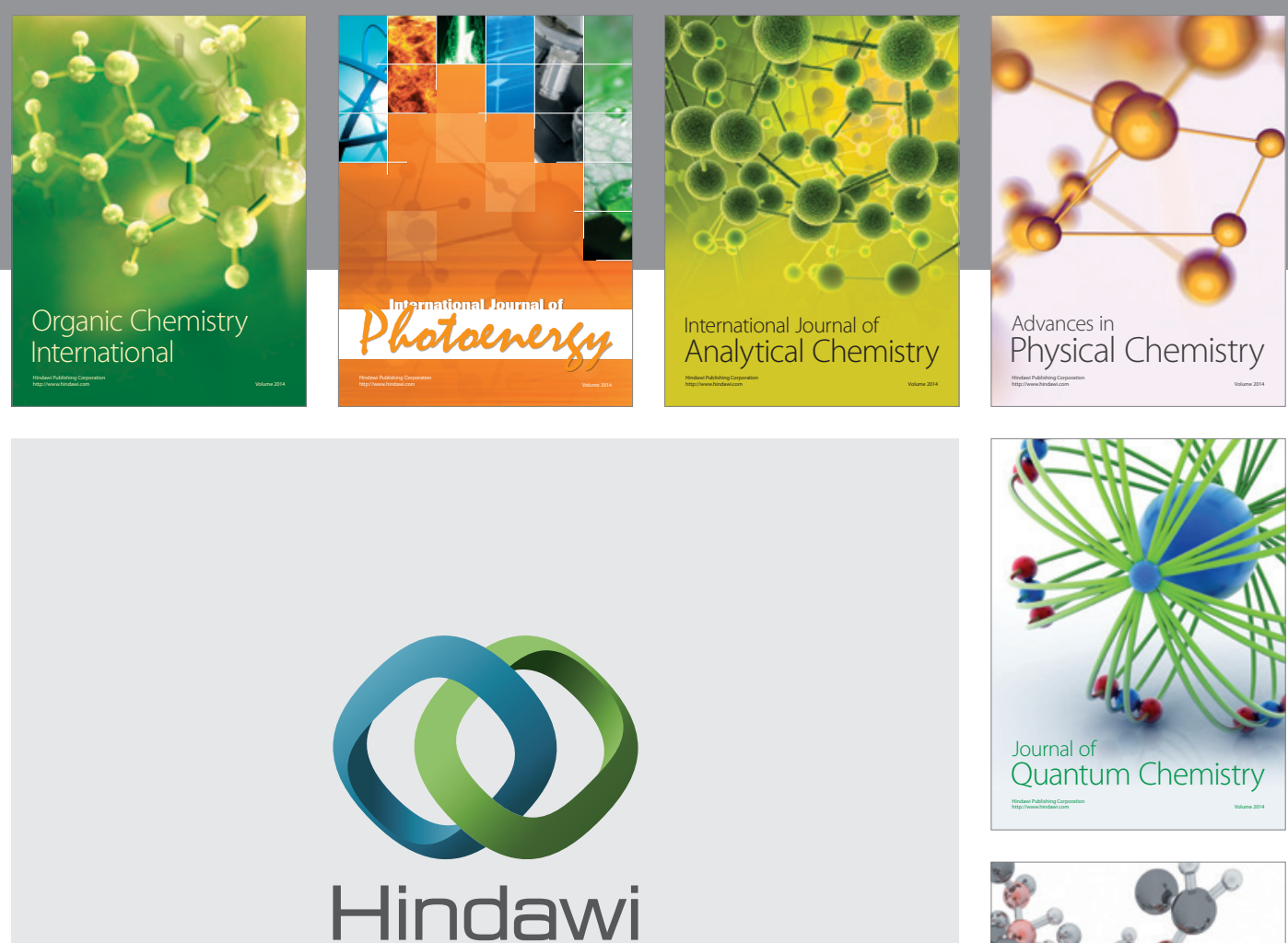

Submit your manuscripts at

http://www.hindawi.com

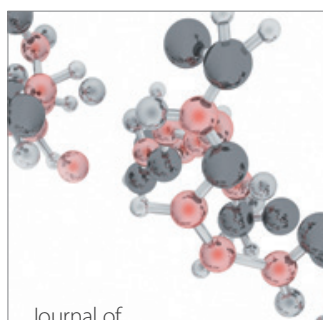

Analytical Methods

in Chemistry

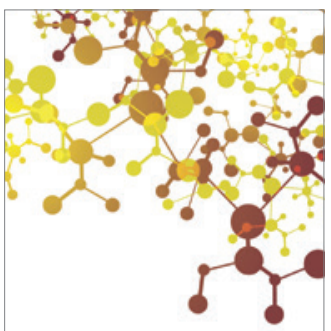

Journal of

Applied Chemistry

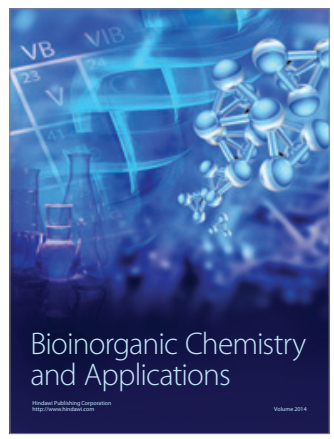

Inorganic Chemistry
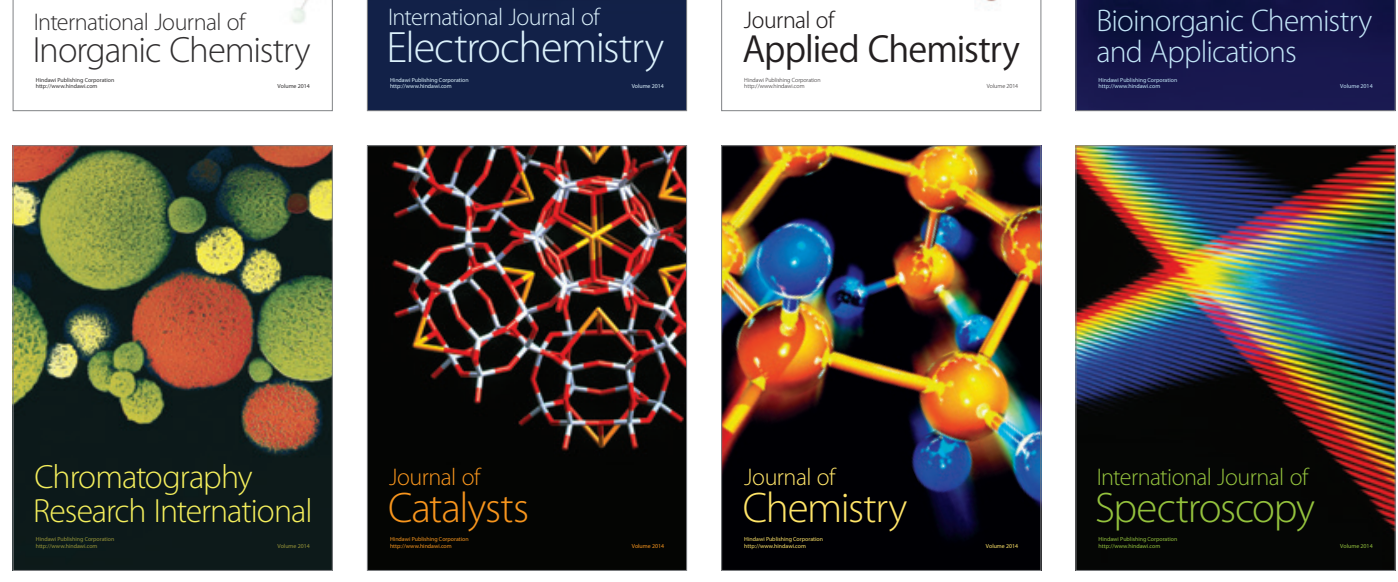